\title{
Operant Behavior of the Rat Can Be Controlled by the Configuration of Objects in an Animated Scene Displayed on a Computer Screen
}

\author{
T. NEKOVÁŘOVÁ, D. KLEMENT \\ Institute of Physiology, Academy of Sciences of the Czech Republic, Prague, Czech Republic
}

Received January 21, 2005

Accepted February 4, 2005

On-line available April 26, 2005

\begin{abstract}
Summary
We developed a novel behavioral task in which rats learn to recognize the configuration of objects in an animated scene displayed on a computer screen. The scene consisted of a moving bar and a stationary rectangle. Rats deprived of food were trained to press a lever for reward in a small chamber located in front of the screen. Lever presses were rewarded only when the bar was at the rectangle. Rats anticipated the reward by gradually increasing frequency of lever pressing as the bar approached the rectangle. Control experiments showed that neither the timing nor the discrimination of rewarded and non-rewarded periods as two discrete conditions explain behavior of the rat. Because the changes in the scene were generated by movement of the object, the presented task could be used for studying neural structures involved in spatial behavior of rats using virtual reality technology.
\end{abstract}

Key words

Configuration recognition $\bullet$ Position recognition $\bullet$ Pattern discrimination $\bullet$ Operant behavior $\bullet$ Virtual reality

\section{Introduction}

Animals assess position and speed of nearby or distant objects, for example, when they are escaping a predator, hunting a prey or when they use celestial bodies for orientation. The representation of position of a moving object is clearly spatial.

Rodent neural structures involved in spatial behavior are mostly studied in tasks in which animals actively move through space (Olton et al. 1978, Barnes 1979, Morris 1981, Rossier et al. 2000, Cimadevilla et al. 2001). Deficit in spatial behavior could then be attributed to a deficit in recognizing subject's position or to a deficit in planning or executing movement towards the target. Few tasks (Klement and Bureš 2000, Paštálková et al. 2003) were designed to test specifically the recognition component of spatial behavior. In experiments of Klement and Bureš (2000), rats were passively transported through an environment. They were trained to recognize certain place by reinforcing lever presses in that location. Paštálková et al. (2003) observed rats watching a rotating scene. They were trained to recognize when the angular displacement of the scene was within a specific sector by reinforcing lever presses in this sector. The presented task follows the task described by Paštálková et al. (2003), but the real scene was replaced 
by a virtual one. The virtual scene has numerous advantages over the real scene. It is not constrained by physical properties of the mechanical experimental apparatus. For example, in the experiments of Pašt'álková et al. (2003) the movement of objects was limited to rotation around a common center, all objects moving with the same angular speed, etc. To overcome these constraints a new apparatus had to be built. On the contrary, virtual reality gives the experimenter almost unlimited control over the shape of objects, their positions, speeds and movement trajectories over the screen. It is possible to change the size of the objects and their mutual spatial relations in ways that would be impossible with physical objects. In addition, virtual reality tasks can be easily applied to primates and humans. This makes it possible to examine humans and rats with the same tasks, therefore to better understand differences in brain functioning across these species and to better predict effects of drugs on cognitive impairment found in human diseases (e.g. Alzheimer's disease, schizophrenia) based on rodent models of these diseases. Before we can use all the possibilities that virtual reality offers, we have to show that rats are able to understand the relations between moving objects on the screen, which was the goal of this paper. Wan et al. (1999) has already shown in a c-fos study that the rat brain responds differently to a familiar and to a novel arrangements of stationary objects displayed on a computer screen.

A computer monitor was successfully used in other experiments carried out on rodents (Sun et al. 1992, Sahgal and Steckler 1994, Gaffan and Eacott 1995, Keller et al. 2000, Bussey et al. 2001, Prusky et al. 2004). In these tasks, with the exception of Sun et al. (1992), animals responded to stationary patterns, objects or complex scenes. In the experiments of Sun et al. (1992), the stimulus on the screen gradually changed its size in some trials. However, the animals were trained to perceive the stimulus as static. To our knowledge no behavioral task for rodents used an animated scene displayed on a computer screen as stimulus and lever pressing as the operational response.

In the present experiment, a fixed sequence of rewarded and non-rewarded periods was used in all sessions. Two groups of rats were trained to press a lever for food. The first group was trained with the computer monitor switched off and it was used to test whether rats can identify the rewarded periods by timing. The second group was initially trained to discriminate light and dark conditions, then to discriminate two discrete patterns and finally to recognize the configuration of objects in an animated scene. The light-dark and the pattern discrimination trainings were added to the experimental protocol to characterize lever pressing when discrete static stimuli indicated rewarded and non-rewarded periods. Attention to the screen was required in the pattern discrimination task but not in the light-dark discrimination task. The results showed that rat's operant behavior can be controlled by the configuration of objects in an animated scene displayed on the computer screen.

\section{Methods}

\section{Subjects}

The subjects $(n=10)$ were male Long-Evans rats 3 months old at the beginning of the experiment. The rats were obtained from the breeding colony of the Institute of Physiology, Czech Academy of Sciences and housed in groups of 2-3 per cage in a temperature-controlled room $\left(21^{\circ} \mathrm{C}\right)$ with a $12: 12$ light/dark cycle. Water was freely available but access to food was restricted to maintain the rats at 90-95 \% of their free feeding weight. All procedures were in accordance with Institutional and NIH guidelines and the directive of the European Communities Council (86/609/EEC).

\section{Apparatus}

The apparatus (Fig. 1A) consisted of a rat chamber, a feeder, a 15-inch monitor and a computer. The rat chamber was $14.5 \mathrm{~cm}$ wide and $24 \mathrm{~cm}$ long. Three walls were opaque and $36 \mathrm{~cm}$ high. The front wall was only $4 \mathrm{~cm}$ high to enable the rat to see the monitor placed $36 \mathrm{~cm}$ away. The rat chamber and the monitor were placed on two $75 \mathrm{~cm}$ high pedestals. The elevation of the chamber prevented rats from escaping over the front wall. During the experiments a black curtain was put over the monitor and the rat chamber to restrict rat's visual field only to the monitor screen. The rat chamber was equipped with a horizontal lever $(2.5 \times 2.5 \mathrm{~cm}$ large, protruding from the left wall $13 \mathrm{~cm}$ above the floor, $4 \mathrm{~cm}$ from the front wall) and with a semicircular hopper (2.5 $\mathrm{cm}$ diameter, located on the right wall $6 \mathrm{~cm}$ above the floor, $4 \mathrm{~cm}$ from the front wall). When activated, the feeder delivered one to three $20 \mathrm{mg}$-pasta pellets to the hopper through a plastic tube. The computer registered lever pressing, activated feeder and displayed graphics on the monitor screen. The software was written by authors in Quick Basic 7 and used 640 x 480 pixels resolution to display stimuli on the monitor. The 15 -inch monitor had 
$31.4 \mathrm{kHz}$ horizontal refresh rate and $59.0 \mathrm{~Hz}$ vertical refresh rate.

\section{Behavioral training}

All sessions except pretraining sessions consisted of 10-s periods when lever pressing was reinforced (rewarded periods) separated by 20 -s or $40-\mathrm{s}$ periods when lever presses were not reinforced (nonrewarded periods). Two different durations of the nonrewarded periods were used to prevent timing as an effective strategy. Three 20 -s and three 40-s nonrewarded periods each followed by the rewarded period were arranged in a fixed sequence (Fig. 1B). We called this sequence "hypercycle". The hypercycle lasted 4 min and repeated 10 times during each 40-min session. The overall duration of the rewarded periods constituted $25 \%$ of a session.

Although we refer to rats' responses as to lever presses, lever releases were recorded and elicited delivery of pellets.

\section{Pretraining}

Naive food-deprived rats were habituated to the rat chamber and then trained in a continuous reinforcement schedule to press the lever to obtain food. Three to ten sessions lasting from 20 to $90 \mathrm{~min}$ were required for the rats to learn to press reliably.

\section{Group T: Temporal learning}

Five rats (group $\mathrm{T}$ ) were trained to press the lever for reward without visual information indicating whether lever presses would be rewarded or not. The monitor was black during the whole session. The rats were trained for 21 sessions.

\section{Group L: Light-dark discrimination, pattern} discrimination and configuration recognition

A different group of rats (group $\mathrm{L}, \mathrm{n}=5$ ) was successively trained to discriminate light and dark conditions (Phase 1), to discriminate two discrete patterns (Phase 2) and finally to recognize the configuration of objects in an animated scene (Phase 3).

Phase 1: Light-dark discrimination: The rewarded periods were indicated by a white pattern occupying $69 \%$ of the screen on black background (see description of Pattern 1 in Phase 2). Black screen indicated the non-rewarded periods (Fig. 1C). The white pattern illuminated the rat chamber to such extent that rats could discriminate light and dark periods without looking at the monitor. The rats were trained for 21 sessions.

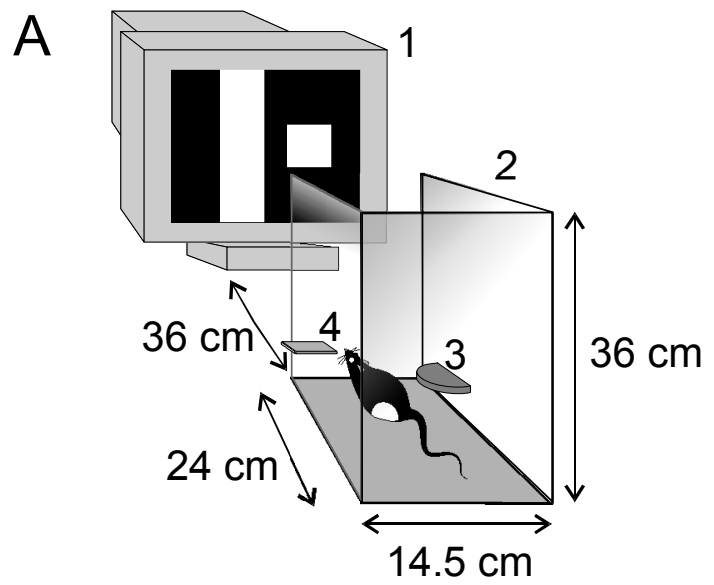

B Hypercycle:

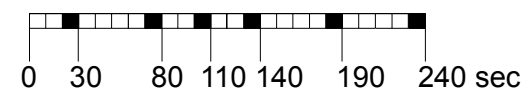

$\square$ non-rewarded period

- rewarded period

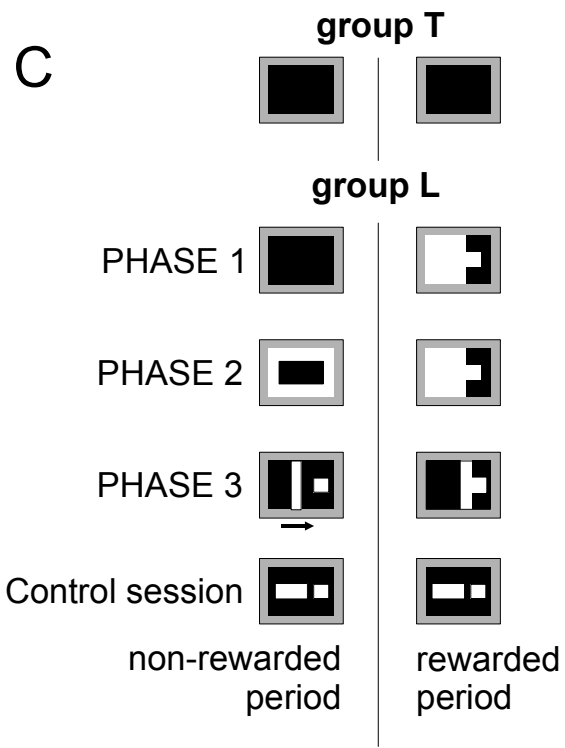

Fig. 1. (A) Scheme of the apparatus: 1 - monitor, 2 - rat chamber, 3 - dipper, 4 - lever. (B) Hypercycle: a fixed sequence of rewarded and non-rewarded periods used in all sessions. The hypercycle repeated 10 times during each $40-\mathrm{min}$ session. (C) Patterns displayed on the computer screen during the rewarded and the non-rewarded periods. Group $T$ tested whether rats can identify the rewarded periods within the hypercycle by timing. Rats in group $L$ were successively trained to discriminate light and dark conditions (Phase 1), to discriminate two discrete patterns (Phase 2) and finally to recognize the configuration of objects in the animated scene (Phase 3 ). During the nonrewarded periods the bar moved from the left side of the screen toward the stationary rectangle. The control session was carried out at the end of Phase 3. 
Phase 2: Pattern discrimination: The rewarded periods were indicated by Pattern 1 (Fig. 1C: Phase 2, the right pattern) and the non-rewarded periods by Pattern 2 (Fig. 1C: Phase 2, the left pattern). Locations [horizontal, vertical] of objects on the screen are given relative to the upper left corner. Pattern 1 consisted of a white rectangle (location: [0, 0] pixels; width $\mathrm{x}$ height: $400 \times 480$ pixels) touching a white square (location: [400, 170] pixels; width $\times$ height: $140 \times 140$ pixels) on black background. Pattern 1 was the same as the pattern displayed on the screen during the rewarded periods in the light-dark discrimination task. Pattern 2 was a black rectangle (location: [102, 170] pixels; width x height: 435 x 220 pixels) on white background. Since the white area of Pattern 2 was equal in size to the white area of Pattern 1, brightness of both patterns was equal. The transition between patterns was achieved by gradual redrawing small squares $(5 \times 5$ pixels) until the new pattern was completed. It lasted 1.2 seconds and it was not accompanied by any change in brightness intensity. The rats could not detect the change of patterns without observing the monitor screen. The rats were trained for 21 sessions.

Phase 3: Recognition of the configuration of objects in an animated scene: During the non-rewarded periods a white vertical bar (width $\mathrm{x}$ height: $100 \times 480$ pixels) moved from the left side of the screen toward a white stationary rectangle (location: [400, 170] pixels; width $\mathrm{x}$ height: $150 \times 140$ pixels) located on the right. When the moving bar touched the rectangle, it stopped there and the non-rewarded period switched to the rewarded period (Fig. 1C). The rewarded period lasted 10 seconds after which the rectangle disappeared and it was redrawn on the left side of the screen. This produced a brief flash. The speed of the movement was 17 pixels per second $(7.5 \mathrm{~mm} / \mathrm{s})$ during 20 -s non-rewarded period and 8.5 pixels per second $(3.75 \mathrm{~mm} / \mathrm{s})$ during $40-\mathrm{s}$ nonrewarded period. The rats were trained for 22 sessions.

A control session was inserted between the $21 \mathrm{st}$ and the 22nd training sessions. A novel stationary pattern common to both rewarded and non-rewarded periods was displayed on the screen (Fig. 1C). The pattern consisted of a large rectangle (location: [60, 180]; width $\mathrm{x}$ height: $320 \times 120$ pixels) and of a small rectangle (location: [440, 170]; width $\mathrm{x}$ height: $150 \times 140$ pixels). The large rectangle was redrawn after the end of the rewarded period. The redrawing produced the same flash as redrawing the moving bar in the sessions with the moving bar.
Analyses

All evaluated parameters were analyzed using one-way or two-way repeated ANOVA measures. The post hoc Newman-Keuls test was used when appropriate. Significance was set at $\mathrm{p}<0.05$. The data are reported as averages \pm S.E.M.

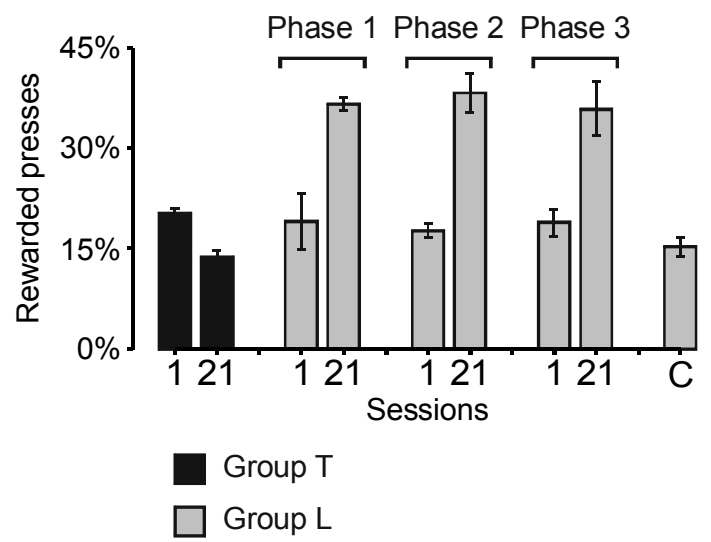

Fig. 2. Preference for the rewarded periods in the beginning (session 1), in the end (session 21) of the training in each task and in the control session. Group T: temporal learning, group L: light-dark discrimination (Phase 1), pattern discrimination (Phase $2)$, recognition of the configuration of objects in the animated scene (Phase 3) and in the control session.

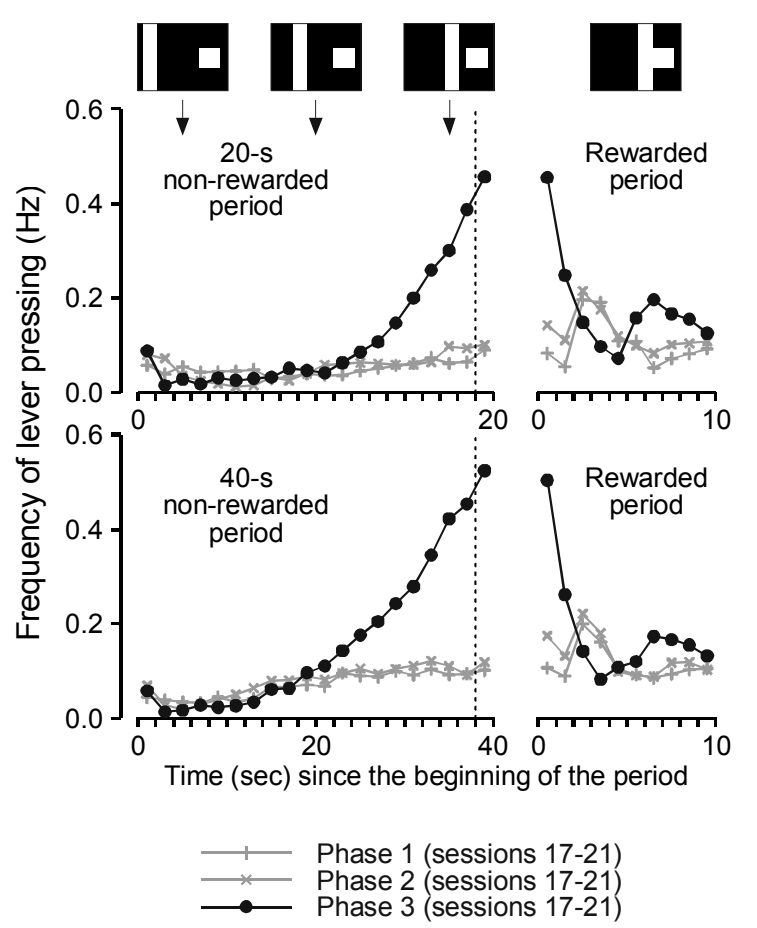

Fig. 3. Distribution of lever presses during the non-rewarded periods and the following rewarded periods in group $L$ at the end of Phases 1,2 and 3. The data are averaged across sessions 17 through 21. The figures above the graphs show position of the moving bar on the computer screen in Phase 3. The vertical dotted line indicates a point at which the distance between the moving bar and the stationary rectangle was equal to $1^{\circ}$ for an observer at $40 \mathrm{~cm}$ distance. The $1^{\circ}$ corresponds to the visual acuity of rats (Birch and Jacobs 1979, Dean 1981, Keller et al. 2000). 


\section{Results}

\section{Percentage of rewarded presses}

Percentage of rewarded presses shows preference for the rewarded periods. In the context of the present experiment this indicates whether the rats recognized the rewarded periods using stimuli on the screen.

The rats in group $\mathrm{T}(\mathrm{n}=5)$ were trained without any visual information indicating whether lever presses would be rewarded or not. During training the percentage of rewarded presses decrease within the first four sessions from $20.4 \pm 0.6 \%$ to $14.9 \pm 0.5 \%$ and it was between $13.7 \%$ and $16.4 \%$. Random lever pressing would result in $25 \%$ of rewarded presses. The lower percentage of rewarded presses than expected is explained by observation that the rats ate pellets immediately after they had been delivered. Number of lever presses increased within the first four sessions from $217 \pm 51$ to $508 \pm 60$ and it was between 456 and 1040 afterwards. Low percentage of rewarded presses compared to both expected value and group L (see below) indicates that timing strategy was not effective for identifying the rewarded periods.

The rats in group $\mathrm{L}(\mathrm{n}=5)$ were initially trained to discriminate light and dark conditions (Phase 1). During training the proportion of rewarded presses increased gradually from $19 \pm 4 \%$ to $37 \pm 1 \%$. Average number of lever presses was between 108 and 266 in all sessions. When the same rats were trained to discriminate two discrete patterns of equal brightness (Phase 2), similarly to Phase 1 , percentage of rewarded presses increased gradually from $18 \pm 1 \%$ to $38 \pm 3 \%$. The average number of lever presses decreased within the first eight sessions to an asymptotic level and it was between 142 and 195 afterwards. In the last phase of the training (Phase 3) the rats were trained to press when the moving bar was touching the stationary rectangle. Percentage of rewarded presses increased from $19 \pm 2 \%$ to $36 \pm 4 \%$. Number of lever presses was between 300 and 575 with a mild decreasing tendency. Only $15 \pm 1 \%$ out of $569 \pm 47$ responses were rewarded in the control session when the same pattern was present during rewarded and nonrewarded periods.

The first sessions from each phase show the performance of untrained animals whereas the $21 \mathrm{st}$ sessions show an asymptotic performance of trained animals. Percentages of rewarded presses in the first sessions, in the 21st sessions of Phases 1, 2 and 3 and in the control session (Fig. 2, group L) were compared by one-way ANOVA with repeated measures $(\mathrm{F}(6,24)=$ 14.52, $\left.\mathrm{p}<10^{-6}\right)$. All the first sessions and the control session were different from all the 21 st sessions $(p<0.001)$. No other differences were found. Thus the rats from group L learned to recognize the rewarded periods using the stimuli on the screen in all three training phases.

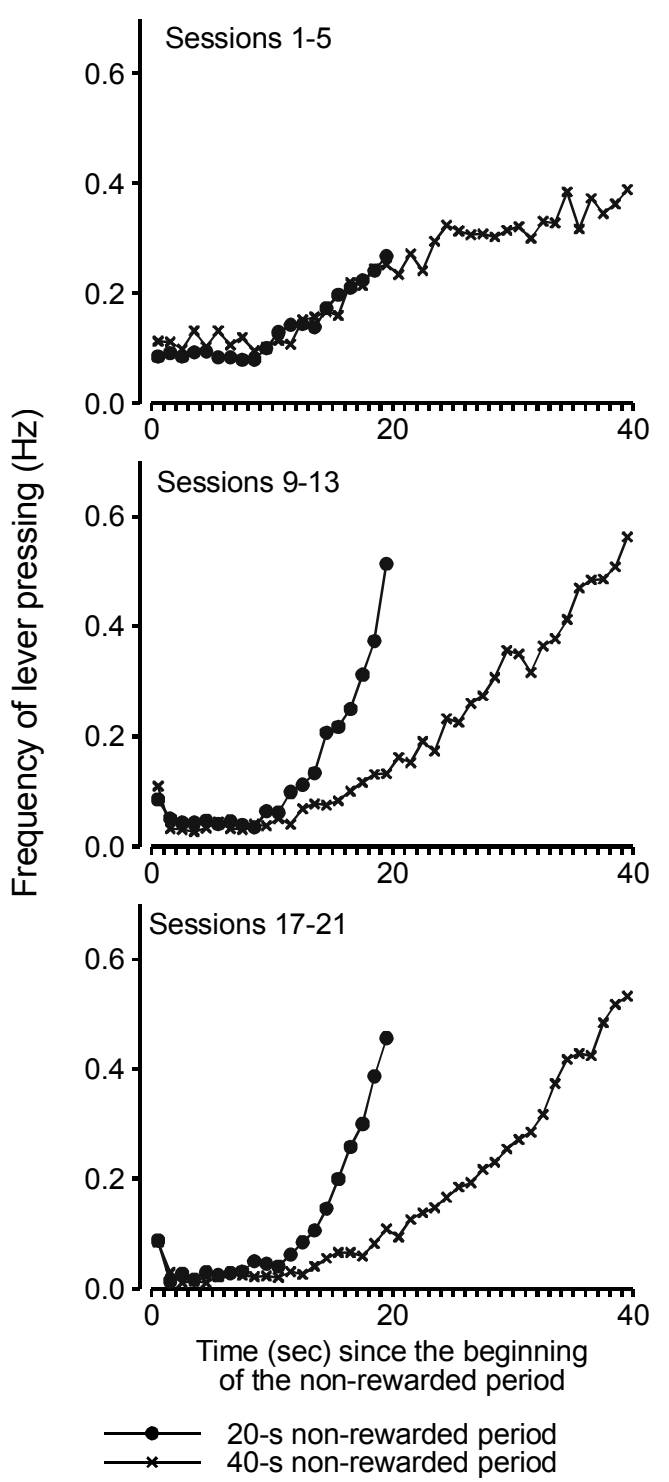

Fig. 4. Development of distribution of lever presses in the nonrewarded periods in Phase 3. Data are averaged across five consecutive sessions.

\section{Distribution of lever presses}

Distribution of lever presses within the nonrewarded periods could reveal whether the rats anticipated the onset of the rewarded periods.

Figure 3 shows frequency of lever pressing during the 20-s non-rewarded period (in 1-s bins), during the 40-s non-rewarded period (in 2-s bins) and during the 
rewarded periods (in 1-s bins) averaged across sessions 17 through 21. The frequency was low in the first half of both non-rewarded periods in all phases. It remained low in Phases 1 and 2 but it rose steeply toward the end in Phase 3. Two-way ANOVA (Phase $\mathrm{x}$ Time bin, $3 \times 20$ ) with repeated measures on both factors showed significant interaction $(20$-s period: $F(38,152)=16.90$, $\mathrm{p}<10^{-6} ; 40$-s period: $\left.\mathrm{F}(38,152)=30.98, \quad \mathrm{p}<10^{-6}\right)$. The following report of post hoc test is restricted to comparison of frequencies among phases at corresponding time bins, it means that we are identifying those time bins in which the frequencies from Phases 1, 2 and 3 differ among themselves (Fig. 3). The frequency was higher in Phase 3 than in Phases 1 and 2 after $14 \mathrm{~s}$ in the 20 -s period $(\mathrm{p}<0.03)$ and after $24 \mathrm{~s}$ in the 40 -s period $(p<0.02)$. No other differences were found. The steep increase in lever pressing corresponded to position of the moving bar (Fig. 3), but not to time elapsed since the beginning or remaining till the end of the non-rewarded period (Fig. 4 - the last graph). The distribution of lever presses during the non-rewarded periods was similar in Phases 1 and 2 but different in Phase 3. The high rate of lever pressing before the end of the non-rewarded periods in Phase 3 indicates that the rats anticipated the onsets of the rewarded periods.

The distribution of presses during the rewarded periods was also different in Phase 3 compared to Phases 1 and 2. The frequency of lever pressing had a single peak with maximum at the third second in Phases 1 and 2 (Fig. 3). In Phase 3 the rate of responding was highest in the very beginning and after a decline it peaked again in the second half of the rewarded period (Fig. 3). Proportion of rewarded periods in which rats did not respond at all was $11.7 \pm 3.7 \%$ in Phase $1,15.4 \pm 3.8 \%$ in Phase 2 and only $5.1 \pm 0.7 \%$ in Phase 3. One-way ANOVA with repeated measures showed significant difference between phases $(\mathrm{F}(2,8)=5.15, \quad \mathrm{p}<0.04$; Phase $3<$ Phase 2, $\mathrm{p}<0.04)$. Percentage of rewarded periods with at least two responses calculated out of rewarded periods in which rats responded was $41.9 \pm 3.4$ $\%$ in Phase $1,27.7 \pm 8.1 \%$ in Phase 2 and $70.9 \pm 5.9 \%$ in Phase 3. One-way ANOVA with repeated measures showed significant differences among phases $(\mathrm{F}(2,8)=18.40, \quad \mathrm{p}<0.001 ; \quad$ Phase $2<$ Phase $1<$ Phase 3 , $\mathrm{p}<0.05$ ). Due to the high rate of responding shortly before the onset of rewarded period in Phase 3, rats receive more reward in Phase 3 and missed fewer rewarded periods than in Phases 1 and 2.

Figure 4 shows the frequency of lever pressing in 1-s bins during the non-rewarded periods at the beginning (sessions 1 to 5), in the middle (sessions 9 to 13) and in the end (sessions 17 to 21) of Phase 3. In the beginning of the training the frequency was stable for the first 10 seconds, then it gradually increased and became stable again for the last $16 \mathrm{~s}$. The 20-s period and the first half of the 40-s period were compared by two-way ANOVA (Period $\mathrm{x}$ Time bin, $2 \times 20$ ) with repeated measures on both factors. The main effect of Time bin was significant $\left(\mathrm{F}(19,76)=11.81, \mathrm{p}<10^{-6}\right)$ but not the main effect of Period $(F(1,4)=0.40, p>0.56)$ and not the interaction $(F(19,76)=1.28, p>0.22)$. In the middle and at the end of the training the frequency was also stable for the first $10 \mathrm{~s}$ but the following increase was steeper in the 20 -s period than in the 40-s period. Two-way ANOVA (Period $\mathrm{x}$ Time, $2 \mathrm{x} 20$ ) with repeated measures on both factors showed significant main effect of Time (sessions 9-13: $\quad F(19,76)=18.06, \quad p<10^{-6} ; \quad$ sessions $17-21$ : $\left.\mathrm{F}(19,76)=25.83, \mathrm{p}<10^{-6}\right)$, significant main effect of Period (sessions 9-13: $\mathrm{F}(1,4)=71.17, \mathrm{p}<0.01$; sessions 17-21: $\mathrm{F}(1,4)=23.11, \quad \mathrm{p}<0.01)$ and significant interaction (sessions 9-13: $\mathrm{F}(19.76)=19.50, \mathrm{p}<10^{-6}$; sessions 17-21: $\left.\mathrm{F}(19,76)=19.68, \mathrm{p}<10^{-6}\right)$. At the beginning of Phase 3 , the increase in frequency of lever pressing during the nonrewarded periods corresponded to the time elapsed since the beginning of the periods. In the middle and at the end of Phase 3 the increase corresponded to the position of the moving bar.

\section{Discussion}

Rats in group $\mathrm{T}$ were trained without a discriminative stimulus on the screen. They emitted high number of responses out of which only small fraction was rewarded. This experiment showed that timing strategy alone was inefficient for identifying rewarded and nonrewarded periods. Rats in group L were trained to recognize rewarded and non-rewarded periods according to the stimuli displayed on the screen. Because the only difference between the experiment done with group $\mathrm{T}$ and experiments done with group $\mathrm{L}$ were the stimuli on the screen, it is possible to interpret the higher percentage of rewarded presses in group L as an ability to recognize the rewarded stimuli. The rats in group $\mathrm{L}$ learned to discriminate two discrete conditions in Phases 1 and 2 as well as to recognize the configuration of objects in Phase 3 because the percentage of rewarded presses increased twice as a result of training in each phase. The percentage of rewarded presses always returned to the baseline level 
when a new phase started (Fig. 2). This indicates that the preference for rewarded periods was dependent on visual stimuli on the screen and not on procedural aspects of the tasks or improved temporal learning with prolonged training.

Lever pressing during the non-rewarded periods showed that the rats used different strategy in Phase 3 than in Phases 1 and 2. The rate of responding highly increased toward the end of the non-rewarded periods in Phase 3 but not in Phases 1 and 2. The increase corresponded to the position of the moving bar. The rats developed this behavior during training because at the beginning the rate corresponded to the time elapsed since the last rewarded period indicating timing strategy (Fig. 4).

Next we discuss possible strategies involved in Phase 3: a) discrimination of moving versus stationary stimulus, b) discrimination of touching versus separated objects, and c) recognition of the spatial relation between the moving objects. Temporal learning has already been discussed above.

Ad a) The rats did not discriminate rewarded and non-rewarded periods by determining whether the bar is moving or not. Otherwise they would be discriminating two discrete conditions and the lever pressing would be the same as in Phases 1 and 2.

Ad b) The rats did not learn to respond when they saw that the bar is touching the rectangle and not to respond when they saw a gap between the two objects. If they were responding in this way, they would increase the frequency of lever pressing when they could not see the gap between the bar and the rectangle. Visual acuity of rats is approximately $1^{\circ}$ (Birch and Jacobs 1979, Dean 1981, Keller et al. 2000). The vertical dotted line in Figure 3 indicates a point at which the distance between the two objects was equal to $1^{\circ}$ for an observer at $40 \mathrm{~cm}$ distance. The rats increased the rate of responding far before this point.

Ad c) The rats reliably increased the rate of responding when they saw stimuli, which were never rewarded. They clearly anticipated the onset of rewarded periods. It is likely that they learned the relation between bar's position and reward availability. The increased rate of responding before the bar touches the rectangle could be explained by an effort to be rewarded as soon as possible. Such behavior is advantageous for the rats. Due to the anticipation the rats missed fewer rewarded periods and received more reward per rewarded period in Phase 3 than they did in Phases 1 and 2. Larger reward received in Phase 3 cannot be explained by a non-specific hyperactivity as the rate of responding during the first half of non-rewarded periods was the same in all phases.

The precise time to reward can be calculated from bar's position and its speed. The rats did not use the speed of the bar to predict time to reward as the anticipation was not a function of time to reward but a function of the bar position only (Figs 3 and 4).

The observed anticipatory increase of lever pressing is similar to the behavior described by Klement and Bureš (2000) and Paštálková et al. (2003). In the report of Klement and Bureš (2000), rats were passively transported with a feeder. Lever presses were reinforced by delivering food only if they were emitted within a small region of the trajectory. Rats increased the rate of responding before they entered the rewarded region. The rate was maximal at the entrance into the rewarded region with a smaller peak at the end. In the experiments of Paštálková et al. (2003), rats watched objects rotating around a common center. Lever presses were only rewarded when the angular displacement of the rotating scene was within a specific sector. Rats increased the frequency of lever pressing before the angular displacement entered the sector. The experiment described in this paper follows the experiments published by Klement and Bureš (2000) and Paštálková et al. (2003). All these experiments used lever pressing as the behavioral response to continuously changing stimuli. In these experiments the changes were generated by movement of the animal or the object(s) through space. It is possible that the neural structures critical for spatial behavior are also critical for the ability to recognize the target configuration of a continuously changing scene. (Klement et al. 2005) showed that hippocampus is critical for preferential lever pressing at a rewarded region when rats are passively transported as they were in experiments of Klement and Bureš (2000). In contrast to the settings described by Klement et al. (2005) the rats do not move through space in the presented task, therefore the critical role of hippocampus has to be tested. The design of the apparatus makes it possible to use the light-dark discrimination task which is independent of hippocampus (Klement et al. 2005) as a control task. Since the only difference between these tasks is the stimulus on the screen, the effect of hippocampal lesion on the ability to recognize a target configuration can be assessed directly.

We believe that the presented task is a step towards using computer tests and virtual reality for studying spatial behavior in rodents. Virtual reality is 
commonly used in human studies (Astur et al. 2004, Daum et al. 1991, Hamilton et al. 2002, Iaria et al. 2003, Scott and Resnick 2002, Kalová et al. 2005). Exposure of rodents and humans to identical tasks would allow a direct comparison of the effects of lesions and drugs. This would help to understand differences in the brain functioning and to better predict effects of drugs on cognitive impairment found in human diseases (e.g.
Alzheimer disease, schizophrenia) based on rodent models of these diseases.

\section{Acknowledgements}

We thank Jan Bureš and Edo Kelemen for their helpful suggestions and comments. This work was supported by GACR grant 309/03/0715, by the research project AVOZ 5011922 and by the project MŠMT ČR 1M0002375201.

\section{References}

ASTUR RS, TROPP J, SAVA S, CONSTABLE RT, MARKUS EJ: Sex differences and correlations in a virtual Morris water task, a virtual radial arm maze, and mental rotation. Behav Brain Res 151: 103-115, 2004.

BARNES CA: Memory deficits associated with senescence: a neurophysiological and behavioral study in the rat. J Comp Physiol Psychol 93: 74-104, 1979.

BIRCH D, JACOBS GH: Spatial contrast sensitivity in albino and pigmented rats. Vision Res 19: 933-937, 1979.

BUSSEY TJ, SAKSIDA LM, ROTHBLAT LA: Discrimination of computer-graphic stimuli by mice: a method for the behavioral characterization of transgenic and gene-knockout models. Behav Neurosci 115: 957-960, 2001.

CIMADEVILLA JM, FENTON AA, BUREŠ J: New spatial cognition tests for mice: passive place avoidance on stable and active place avoidance on rotating arenas. Brain Res Bull 54: 559-563, 2001.

DAUM I, SCHUGENS MM, CHANNON S, POLKEY CE, GRAY JA: T-maze discrimination and reversal learning after unilateral temporal or frontal lobe lesions in man. Cortex 27: 613-622, 1991.

DEAN P: Visual pathways and acuity hooded rats. Behav Brain Res 3: 239-271, 1981.

GAFFAN EA, EACOTT MJ: A computer-controlled maze environment for testing visual memory in the rat. $J$ Neurosci Methods 60: 23-37, 1995.

HAMILTON DA, DRISCOLL I, AND SUTHERLAND RJ: Human place learning in a virtual Morris water task: some important constraints on the flexibility of place navigation. Behav Brain Res 129: 159-170, 2002.

IARIA G, PETRIDES M, DAGHER A, PIKE B, BOHBOT VD: Cognitive strategies dependent on the hippocampus and caudate nucleus in human navigation: variability and change with practice. J Neurosci 23: 5945-5952, 2003.

KELLER J, STRASBURGER H, CERUTTI DT, SABEL BA: Assessing spatial vision - automated measurement of the contrast-sensitivity function in the hooded rat. J Neurosci Methods 97: 103-10, 2000.

KLEMENT D, BUREŠ J: Place recognition monitored by location-driven operant responding during passive transport of the rat over a circular trajectory. Proc Natl Acad Sci USA 97: 2946-2951, 2000.

KLEMENT D, PAŠŤÁLKOVÁ E., FENTON AA: Tetrodotoxin infusions into the dorsal hippocampus block nonlocomotory place recognition. Hippocampus 15: 460-471, 2005.

MORRIS RG: Spatial localization does not require the presence of local cues. Learning Motivation 12: 239-260, 1981.

OLTON DS, WALKER JA, GAGE FH: Hippocampal connections and spatial discrimination. Brain Res 139: 295-308, 1978.

PAŠŤÁLKOVÁ E, KELEMEN E, BUREŠ J: Operant behavior can be triggered by the position of the rat relative to objects rotating on an inaccessible platform. Proc Natl Acad Sci U S A 100: 2094-2099, 2003.

PRUSKY GT, DOUGLAS RM, NELSON L, SHABANPOOR A, SUTHERLAND RJ: Visual memory task for rats reveals an essential role for hippocampus and perirhinal cortex. Proc Natl Acad Sci USA 101: 5064-5068, 2004.

ROSSIER J, KAMINSKY Y, SCHENK F, BUREŠ J: The place preference task: a new tool for studying the relation between behavior and place cell activity in rats. Behav Neurosci 114: 273-284, 2000.

SAHGAL A, STECKLER T: TouchWindows and operant behaviour in rats. J Neurosci Methods 55: 59-64, 1994.

SCOTT DM, RESNICK SM: Effect of age on virtual environment place navigation and allocentric cognitive mapping. Behav Neurosci 116: 851-859, 2002. 
SUN HJ, CAREY DP, GOODALE MA: A mammalian model of optic-flow utilization in the control of locomotion. Exp Brain Res 91: 171-175, 1992.

KALOVA E, VLCEK K, JAROLIMOVA E, BURES J. Allothetic orientation and sequential ordering of places is impaired in early stages of Alzheimer's disease: corresponding results in real space tests and computer tests. Behav Brain Res 159: 175-186, 2005.

WAN H, AGGLETON JP, BROWN MW: Different contributions of the hippocampus and perirhinal cortex to recognition memory. J Neurosci 19: 1142-1148, 1999.

\section{Reprint requests}

Daniel Klement, Institute of Physiology, Academy of Sciences of the Czech Republic 1083 Vídeňská, 14220 Prague, Czech Republic. E-mail: dklement@biomed.cas.cz 